Ann. Biol. anim. Bioch. Biophys., 1977, 17 (4), 637-643.

\title{
Résultats obtenus chez la truite Arc-en-ciel concernant l'absorption du glycocolle par l'intestin moyen et par l'intestin postérieur : rôle du sodium dans les différences observées
}

par G. BOGÉ, A. RIGAL, G. PÉRÈS

Institut Michel Pacha,

Laboratoire marifime de Physiologie,

83500 Tamaris $\mathrm{s} / \mathrm{mer}$.

Summary. Middle and distal intestinal absorption of glycocolle in trout. Role of sodium in the differences observed.

The trout intestine is divided into two different parts, the middle and the distal intestine. In this report, we study in vitro and in vivo absorption of ${ }^{14} \mathrm{C}$ glycocolle $(0.5 \mathrm{mM})$ to determine if some functional characteristics of aminoacid uptake are related to these morphological differences in trout.

In vitro as well as in vivo, the amounts of aminoacids absorbed in the distal intestine are always higher than in the middle intestine when media contain $150 \mathrm{mEq} \mathrm{Na}$. In contrast, when sodium is replaced by mannitol, absorption does not differ.

L'intestin de la truite se divise en deux parties morphologiquement distinctes qui sont appelées par Bertin (1958) intestin moyen et intestin postérieur.

Le but de ce travail est de vérifier si des particularités fonctionnelles dans les capacités de transport de l'intestin sont associées à ces différences morphologiques. Nous avons étudié, in vitro et in vivo, l'absorption, par ces différents segments, d'un acide aminé : le glycocolle qui peut faire l'objet d'un transport actif chez de nombreux poissons notamment chez la truite (Bogé, 1972).

\section{Matériel et méthodes.}

Il a été utilisé des truites de $180 \mathrm{~g}$ environ, stockées à $14^{\circ} \mathrm{C}$ et régulièrement alimentées.

Technique d'étude in vitro de l'absorption infestinale. - La technique in vitro est celle de l'intestin retourné de Wilson et Wiseman (1954) appliquée à la truite (Bogé, Rigal ef Pérès, 1975). 
Technique d'éfude in vivo de l'absorption intestinale. - Nous avons eu recours à la technique de perfusion de l'intestin décrite chez la rascasse (Pérès, Rigal et Bogé, 1973).

La perfusion est effectuée à $14^{\circ} \mathrm{C}$ pendant $30 \mathrm{mn}$ chez le poisson non anesthésié et porteur de catheters souples. Le milieu de perfusion est analogue au milieu « in vitro $\gg$.

\section{Résultats.}

I. Etude du passage intestinal du glycocolle.

\subsection{Efude in vitro.}

Nous avons déterminé, après $30 \mathrm{mn}$ les quantités de radioactivité absorbées par la muqueuse de l'intestin entier (absorption muqueuse), la radioactivité retenue à l'intérieur de l'ensemble des tissus de l'intestin (rétention tissulaire) ainsi que celles du milieu séreux (transfert séreux) (fig. 1).

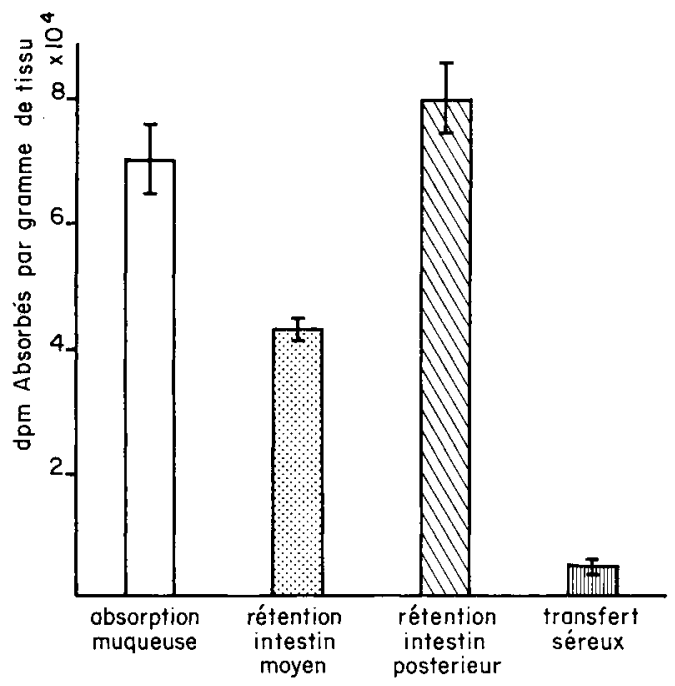

FIG. 1. - Etude in vitro de l'absorption du glycocolle par l'infestin de la truite Arc-en-Ciel et de la radioactivité accumulée dans l'intestin moyen, l'intestin postérieur et le milieu séreux. (Technique des sacs retournés. Durée d'incubation $=30 \mathrm{mn}$ à $20^{\circ} \mathrm{C}$ ).

Concentration initiale en glycocolle $=0,5 \mathrm{mM}$.

RAS $=5,55 \times 10^{4} \mathrm{dpm} / \mu \mathrm{M}$ glycocolle.

La plus grande partie de la radioactivité est retenue dans les tissus intestinaux ; le transfert séreux ne représente qu'une faible proportion de la quantité totale absorbée. La répartition de la radioactivité est inégale le long de l'intestin : la radioactivité de l'intestin postérieur est plus forte que celle de l'intestin moyen. Cependant, elle est toujours supérieure à celle du milieu d'incubation. 
1.2 Efude in vivo.

Les résultats obtenus in vivo, concernant l'absorption de $0.5 \mathrm{mM}$ de ${ }^{14} \mathrm{C}$ glycocolle, sont rassemblés dans la figure 2.

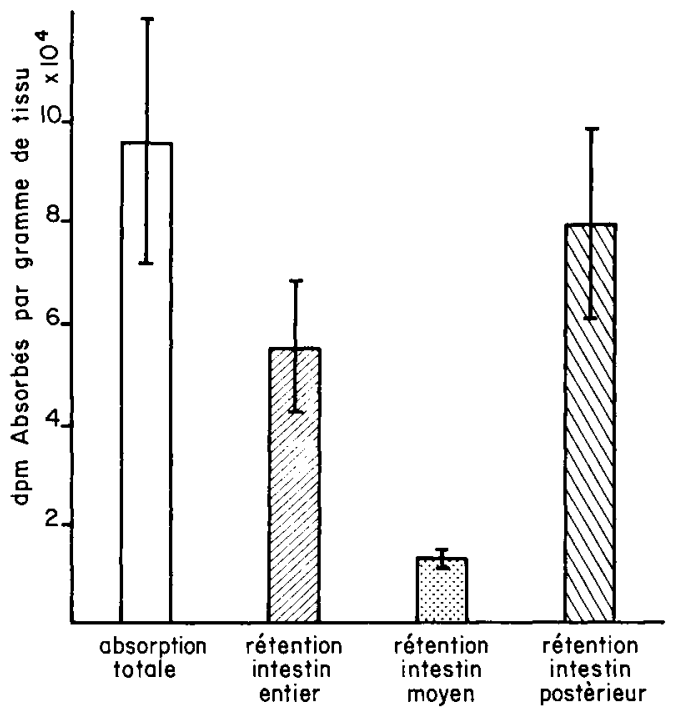

FIG. 2. - Elude in vivo de l'absorption totale du glycocolle par l'intestin de la truife Arc-en-Ciel el de la radioactivité accumulée dans l'intestin entier, l'intestin moyen et l'intestin postérieur. (Technique des perfusions. Durée de la perfusion $=30 \mathrm{mn}$. Température de l'eau $=14^{\circ} \mathrm{C}$ ).

Concentration initiale en glycocolle $=0,5 \mathrm{mM}$.

RAS $=5,55 \times 10^{4} \mathrm{dpm} / \mu \mathrm{M}$ glycocolle.

Après $30 \mathrm{mn}$ de perfusion, la radioactivité résiduelle de l'intestin représente 56 p. 100 de la quantité totale absorbée. De plus, comme in vitro, la radioactivité tissulaire esł beaucoup plus forte dans l'intestin postérieur que dans l'intestin moyen.

Nous voulions savoir si le gradient de rétention tissulaire du glycocolle ne résultait pas simplement d'une plus grande quantité du tissu muqueux ou d'une plus forte incorporation du glycocolle dans les protéines de l'intestin postérieur. Pour ce faire, nous avons isolé par raclage de chaque partie de l'intestin, les tissus muqueux des tissus musculaires dans l'intestin moyen comme dans l'intestin postérieur les premiers représentent 55 p. 100 du poids total.

D'autre part, nous avons évalué la radioactivité des protéines en fin d'incubation ou de perfusion après extraction des lipides à l'aide de chloroforme/méthanol (V/V) ef des petites molécules acido-alcoolo solubles (TCA 5 p. 100 puis alcool éthylique). Le résidu final contenant les protéines est dissous dans $\mathrm{NaOH} 1 \mathrm{~N}$, puis soumis au comptage.

L'incorporation de la radioactivité dans les protéines ne constitue qu'un très faible pourcentage de l'activité totale des tissus (tabl. 1).

In vitro, cette incorporation revêt la même importance dans les deux parties de 
l'intestin. In vivo, elle est sensiblement plus forte dans l'intestin moyen. En conséquence, il n'apparaît pas que l'on puisse rattacher les différences d'incorporation du glycocolle au niveau de l'intestin moyen et de l'intestin postérieur, à des inégalités dans la répartition pondérale du tissu absorbant, ni à l'utilisation du glycocolle pour la synthèse des protéines endogènes de l'organe.

\section{TABLEAU 1}

Etude de la radioactivité incorporée dans les protéines de l'intestin moyen et de l'intestin postérieur, après $60 \mathrm{mn}$ d'incubation in vitro ou de perfusion in vivo (les résultats sont exprimés en p. 100 de l'activité totale incorporée dans le tissu).

\begin{tabular}{lcc}
\hline & Intestin moyen & Intestin postérieur \\
\hline In vifro $\ldots \ldots \ldots \ldots \ldots$ & 3,39 p. 100 & 2,97 p. 100 \\
\hline In vivo $\ldots \ldots \ldots \ldots$ & 15,38 p. 100 & 6,94 p. 100 \\
\hline
\end{tabular}

II. Etude des effets de la suppression du Na du milieu sur les vitesses d'absorption du glycocolle.

Afin d'apprécier l'importance du transport actif dans l'intestin moyen el dans l'intestin postérieur, nous avons comparé les vitesses d'absorption en présence et en

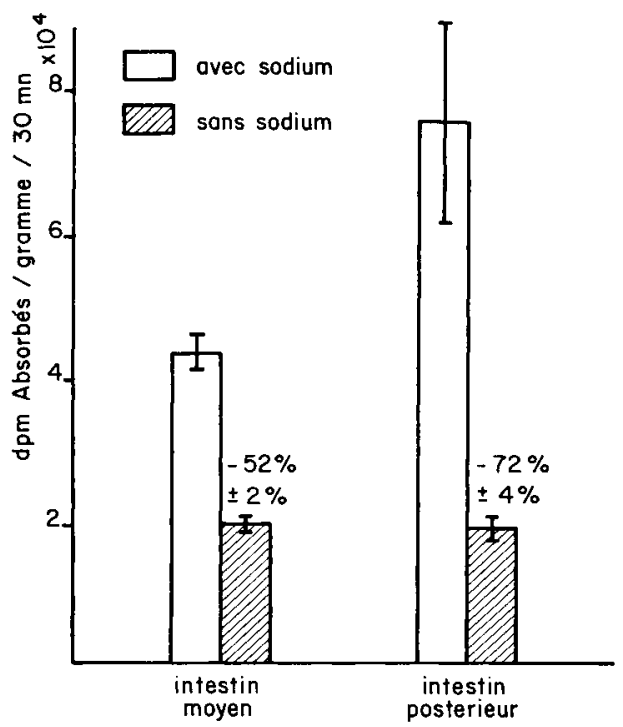

FIG. 3. - Effet du sodium sur l'absorption in vitro du glycocolle $(0,5 \mathrm{mM})$ par l'intestin moyen et l'intestin postérieur de la truite Arc-en-Ciel. Les pourcentages d'inhibition sont indiqués sur la figure. (Technique des «bandes ». Durée d'incubation $=30 \mathrm{mn}$ à $20^{\circ} \mathrm{C}$ ).

Concentration initiale en glycocolle $=0,5 \mathrm{mM}$.

RAS $=5,55 \times 10^{4} \mathrm{dpm} / \mu \mathrm{M}$ glycocolle. 
l'absence de sodium (le $\mathrm{Na}$ étant remplacé par des quantités osmotiquement équivalentes de mannitol). En raison des faibles quantités de radioactivité transportées dans le milieu séreux, nous avons, in vitro, eu recours à la technique des bandes d'intestin (Crane et Mandelstam, 1960).

Deux bandes sont découpées sur chaque partie de l'intestin, l'une étant incubée en présence de sodium, l'autre en l'absence de sodium. Après $30 \mathrm{mn}$, on procède à la défermination des quantités de glycocolle $(0,5 \mathrm{mM})$ qui ont été incorporées par le tissu (fig. 3).

En présence de $\mathrm{Na}$, l'intestin postérieur incorpore plus de glycocolle $(0,5 \mathrm{mM})$ que l'intestin moyen. Cette différence est très fortement réduite lorsque le sodium est remplacé par du mannitol.

In vivo, nous avons réalisé la même étude au moyen de la technique de perfusion en présence ef en l'absence de $\mathrm{Na}$ (fig. 4).



FIG. 4. - Effet du sodium sur l'absorption in vivo du glycocolle $(0,5 \mathrm{mM})$ por l'intestin moyen et l'iniestin postérieur de la truite Arc-en-Ciel. Les pourcentages d'inhibition sont indiqués sur la figure. (Technique des perfusions d'une durée de $30 \mathrm{mn}$ à $14{ }^{\circ} \mathrm{C}$ ).

Concentration initiale en glycocolle $=0,5 \mathrm{mM}$.

RAS $=5,55 \times 10^{4} \mathrm{dpm} / \mu \mathrm{M}$ glycocolle.

En présence de $\mathrm{Na}$, l'absorption du glycocolle est beaucoup plus forte dans l'intestin postérieur. Les différences antéro-postérieures sont encore plus accusées qu'in vitro. Lorsque le $\mathrm{Na}$ est supprimé du milieu, les vitesses d'absorption sont fortement réduites dans les deux parties de l'intestin. Les radioactivités absorbées alors par chaque segment d'intestin sont très voisines. 


\section{Discussion et conclusions.}

1. Ces résultats indiquent qu'il existe le long de l'intestin de la truite un gradient de transport du glycocolle, l'intestin postérieur absorbant plus d'acide aminé que l'intestin moyen.

2. Le gradient d'absorption du glycocolle est associé à un gradient parallèle de rétention tissulaire. Nous avons montré qu'après $30 \mathrm{mn}$ d'incubation, ou de perfusion in vivo, la radioactivité de l'intestin postérieur est largement supérieure à celle de l'intestin moyen. In vitro, ce résultat est dô à un très faible transfert séreux, la majeure partie du glycocolle absorbé étant retenue dans la muqueuse et les tissus sous-jacents. In vivo, une partie importante de la radioactivité absorbée est également fixée dans l'intestin, principalement dans l'intestin postérieur.

3. Dans une précédente communication (Bogé, Rigal ef Pérès, 1975), nous avons montré que la distinction régionale dans les vitesses d'absorption du glycocolle était en relation avec les caractéristiques de saturation des mécanismes de transport par des quantités croissantes d'acide aminé. Les résultats présentés indiquent qu'en outre, ces différences sont en rapport avec la présence dans les milieux de quantités importantes de $\mathrm{Na}(150 \mathrm{mEq})$.

Ces résultats suggèrent que chez la truite, comme chez le rat, le gradient d'absorption des acides aminés est lié à l'activité des mécanismes actifs. En effet, le sodium est indispensable au déroulement du transport actif de nombreux solutés (Crane, 1965 ; Curran et al., 1967).

Les différences concernant les mécanismes actifs assurant le transport du glycocolle dans l'intestin moyen et dans l'intestin postérieur confirment les premières observations de Yamamoto (1966) faisant état d'une spécialisation fonctionnelle des deux régions de l'intestin de la truite.

Commission CNERNA Digestion-Absorption, Tours, 13 novembre 1976.

\section{Références}

BERTIN L., 1958. L'appareil digestif des agnathes ef des poissons, 1268-1273. In Grassé P. P. Traité de Zoologie. Masson et Cie, Paris.

BOGÉ G., 1972. Recherches sur la nature ef les caractéristiques de l'absorption in vifro du glycocolle et du sodium par l'intestin de la truite Arc-en-ciel (Salmo gairdnerii). Thèse Doctorat $3^{\mathrm{e}} \mathrm{Cycle}$, Lyon.

BOGÉ G., RIGAL A., PÉRÈS G., 1975. Recherches sur l'absorption intestinale du glycocolle chez la truite (Salmo gairdnerii). J. Physiol., 71, 265 A.

CRANE R. K., 1965. Na-dependent transport in the intestine and other animal tissues. Fed. Proc., 24, 1000-1006.

CRANE R. K., MANDELSTAM P., 1960. The active transport of sugar by various preparations of: hamster intestine. Biochim. biophys. Acta, 45, 460-476.

CURRAN P. F., SCHULTZ S. G., CHEZ R. A., FUISZ R. E., 1967. Kinetics relations of the Na amino acid interaction of the mucosal border of the intestine. J. gen. Physiol., 50, 1261. 
PÉRĖS G., RIGAL A., BOGÉ G., 1973. Description el discussions comparatives de deux méthodes usuelles d'absorption intestinale in vivo appliquées au poisson. Ann. Insf. Michel Pacha, 6, 18-25.

WILSON T. H., WISEMAN G., 1954. The use of everted small intestine for the study of the transference substances from the mucosal to serosal surface. J. Physiol., G. B., 123, 116-125.

YAMAMOTO T., 1966. An electro microscope study of the columnar epithelial cell in the intestine of fresh water teleost : gold fish (Carassius auratus) and rainbow trout (Salmo irideus). $Z$. Zellforsch. Mikr. Anat., 72, 66-87. 